\title{
Keeping Pace with Our Changing Times
}

\author{
Dorothy S. Becvar
}

Published online: 4 August 2011

(C) Springer Science+Business Media, LLC 2011

Perhaps needless to say, it is the job of a professional journal to help its readers stay abreast both of developments in the larger society as well as of updated information and internal innovations that are likely to have an impact on those served by the members of the targeted group. Certainly as marriage and family therapists (MFTs), along with other related mental health professionals, it is essential that we be well informed and able to respond to our clients in ways that are sensitive to whatever new or old challenges they may be facing. To that end, in this issue we offer articles that focus on three such challenges: the increasing number of military marriages and families experiencing deployment; the ongoing and ever-present need to understand relational dynamics; and the growing awareness of and sensitivity to multicultural issues and the need for competence in this area.

Since the terrorist attacks of 09/11/01, more and more service members have been called to active duty. As we are increasingly likely to be working with military marriages and families we are called upon to understand both their strengths and their areas of need. In an article titled "Military Marriages: The Aftermath of Operation Iraqi Freedom (OIF) and Operation Enduring Freedom (OEF) Deployments" authors Joyce Baptist, Yvonne Amanor-Boadu, Kevin Garrett, Briana Nelson Goff, Jonathon Collum, Paulicia Gamble, Holly Gurss, Erin Sanders-Hahs, Lizette Strader, and Stephanie Wick describe a qualitative study revealing deployment-related challenges as well as aspects of resilience experienced by members of the military and their families. In the second article on this topic, "Military Marriages: The Aftermath of Operation Iraqi Freedom (OIF) and Operation Enduring Freedom (OEF) Deployments", Glenn Hollingsworth provides a framework for intervention with couples who have experienced the challenges of deployment.

The second topic, relationship dynamics, is of course fundamental to the practice of marriage and family therapy, and probably one that we will never fully understand in terms of its nuances and complexity. Nevertheless, explorations in this area may continue to enhance our knowledge and, hopefully, our effectiveness. In the article titled "Relational Financial Therapy: An Innovative and Collaborative Treatment Approach", Ji-Hyun Kim,

D. S. Becvar $(\bowtie)$

Saint Louis University, Saint Louis, MO, USA

e-mail: becvards@slu.edu 
Gerry Gale, Joseph Goetz, and Maria Bermùdez describe an approach to dealing with financial issues faced by couples using co-therapy teams of MFTs and financial planners. This article is followed by two quantitative studies with implications for couples. In the first, "Tracking Marital Adjustment, Hostility, and Physical Functioning Across Time in a Therapy Population: A Biopsychosocial Model” by Nathan Wood, Russell Crane, and Peggy Keller, various factors related to marital satisfaction and adjustment are explored and described. In the second, "Getting to the Root of Relationship Attributions: Family-ofOrigin Perspectives on Self and Partner Views" by Brandon Burr, Brandt Gardner, Dean Busby and Sarah Lyon, the focus is on the impact one's family of origin has on attributions made later by couples about themselves and each other.

The third topic, multicultural issues, continues to grow in significance given an increasing awareness of and openness to sexual diversity as well as the changing demographics both in our society and in the global community. Four qualitative studies offer interesting insights relative to this important topic. First, Markie Blumer and Megan Murphy provide an article titled, “Alaskan Gay Males' Couple Experiences of Societal Non-Support: Coping Through Families of Choice and Therapeutic Means" in which they explore both the societal experiences and the coping mechanisms of their participants. The next article, "Family Dynamics and Changes in Sibling of Origin Relationship After Lesbian and Gay Sexual Orientation Disclosure" by Angela Hilton and Dawn Szymanski, sheds light on the experiences of heterosexual biological siblings of lesbians and gay males following disclosure by the latter of their sexual orientation. Shifting to another aspect of multiculturalism, the third article in this section, "Approaching the "Resistant:" Exploring East Asian International Students' Perceptions of Therapy and Help-Seeking Behavior Before and After They Arrived in the United States" by Hao-Min Chen and Denise Lewis, provides a consideration of six East Asian international students regarding their perceptions of therapy. Finally, in the article titled "Meeting a New Me: An Autoethnographic Journey into Kenya and Back" by Miranda Gilmore and Rajeswari Natrajan-Tyagi, we are offered an exploration of the impact of the experience of living in a foreign culture and then returning to one's native country.

Whether the world really is changing more rapidly than it has in the past, or this just seems to be the case given the sophisticated technology that enables us to have moment to moment awareness of what is happening across the globe, ours is a fast-paced context that requires us to be able to respond continually to ever changing news of difference. Included in this charge are both the professionals who serve clients and the journals that serve professionals by helping them to stay well-informed. My hope and belief is this issue, as well as those that have preceded it and those that will follow, have, will, and do just that. 